\title{
Do Voting Power Considerations Explain the Formation of Political Coalitions? A Re-evaluation
}

\author{
Vincent C. H. Chua
}

Singapore Management University, Singapore

(eMail: vincentchua@smu.edu.sg)

Dan S. Felsenthal

University of Haifa, Israel

(eMail: dfelsenthal@poli.haifa.ac.il)

\begin{abstract}
In this paper, we view the party charged with forming a governing coalition immediately following an election as attempting to put together a coalition that will, with some compromise, promote its ability to implement its legislative agenda and to influence the legislative outcome in this direction. We thus view the problem of the coalition leader as one of maximizing its influence as measured by the Penrose measure of absolute voting power and subject this hypothesis and three variants to empirical testing using election data from nine countries. Two variants, namely: restricting the maximization process to the set of closed winning coalitions, or likewise but with a further requirement that the winning coalition selected be of minimal range, achieved levels of predictive success comparable to the Leiserson-Axelrod minimal range theory, suggesting that a closer examination of the role of a priori measures of power in political coalition formation may be useful.
\end{abstract}

Keywords coalition formation, closed minimal range theory, indices of power, voting power

\section{Introduction}

In a multi-party system in which no single party controls a decisive majority in the legislature, the party charged with forming a new government sometimes has the option of governing as a minority government. Such a course 
of action will preserve the party's control over all ministerial portfolios and its legislative agenda but it will also mean an arduous uphill battle to see the agenda through since, as a governing party, it does not control the required majority in the legislature. Therefore, in many instances, forming a majority coalition government ${ }^{1}$ is a preferred arrangement. Here, it will usually have the liberty to decide which other party or parties may join the governing coalition.

Involving other parties in a coalition government, however, entails a cost and while the leading party still has control over the distribution of ministerial portfolios and plays the key role in shaping the legislative agenda, it will have to cede some measure of control to other coalition members. The vulnerability of the governing coalition to defection will also feature in the political calculations. Citing these considerations in a 1995 interview, Aumann (see van Damme, 1998) hypothesized that, when selecting its coalition partners, a coalition leader will act in a manner that will maximize its Shapley (1953) value. ${ }^{2}$

In Chua and Felsenthal (2008), we subjected this hypothesis and three variants to empirical testing using two sets of data: historical election data tabulated in de Swaan (1973) for eight European countries and for Israel; and detailed election data covering all Israeli elections from 1949 through 2006. In this connection, consistent with Aumann's elaboration of his hypothesis, we defined a winning coalition as any coalition that controls a simple majority of seats in the legislature and which included the party charged with forming the coalition. But, as our investigation revealed, neither the hypothesis nor its variants managed a level of predictive success that is anywhere near that achieved by the closed minimal range theory ${ }^{3}$ or, for that matter, by the minimum size principle, ${ }^{4}$ leading us to summarily reject the hypothesis and its variants.

The behavioral considerations that led to Aumann's hypothesis were, however, intuitive and compelling and have long been the subject of inquiry by political scientists who made a clear distinction between those considerations that are 'office-seeking' and those that are 'policy-seeking.' In advocating the

\footnotetext{
${ }^{1}$ In this paper, the term coalition is regarded as synonymous with the term alliance in the sense of Felsenthal and Machover $(2002,2008)$ and should be understood as such.

${ }^{2}$ In the context of simple voting games, the Shapley value is usually referred to as the ShapleyShubik index. See Shapley and Shubik (1954).

${ }^{3}$ Briefly, on a policy scale in which any two adjacent parties are regarded as one unit distance apart, the range of a coalition is the distance between the two parties in the coalition whose positions on the policy scale are furthest apart. The minimal range theory, due to Leiserson (1966) and to Axelrod (1970), hypothesizes that the coalition formed will be of minimal range.

${ }^{4}$ This hypothesis is due to Riker $(1959,1962)$ and to Gamson $(1961)$. Gamson uses the term cheapest winning coalition to describe the winning coalition controlling the smallest total number of seats (or votes) while Riker uses the term coalition of minimal size.

${ }^{5}$ For a discussion of these different motivations, the reader is referred to Laver and Schofield
} 
Shapley value as the relevant summary measure for the different behavioral considerations, Aumann had implicitly emphasized the office-seeking element, the element that is associated with the notion of power as prize or $P$ power. ${ }^{6}$ As explained in Felsenthal and Machover (1998: 18), the use of such a measure implicitly regards the coalition formation process as an $n$-person bargaining game involving a fixed purse - the prize of power. Political parties with representation in the legislature are, from this perspective, viewed as participating in this bargaining game with the winning coalition capturing the entire pie which it then proceeds to divide among its members.

But, in an actual legislature, it is unclear if this office-seeking motive indeed occupies center-stage position in the 'mind' of the party charged with forming the government, and even when it does feature in an important way, whether it is not merely because control over key political portfolios is instrumental to the smooth implementation of the party's policy agenda. After all, when a party is voted into power, it is usually based on the merits of the party platform and on the premise that there is a good chance the party will deliver on its promises, which in turn puts pressure on the party to perform. From a realist perspective, therefore, moving the party agenda forward and/or influencing the legislative outcome in this direction would seem to be a more natural and central pre-occupation of any party leading the coalition formation process. Additionally, this desired influence ought to be over the period of its tenure in office, highlighting a certain permanency in the arrangement that is being sought. ${ }^{7}$ To differentiate this type of coalitional arrangement from those that are one-off or of an ad hoc nature, Felsenthal and Machover (2008) have aptly termed such arrangements alliances.

In an alliance, the leading party is viewed as participating in a composite voting game, one involving members within the governing coalition and the other involving the entire legislature, and the influence that the leading party has in the legislature is the product of its influence within the governing coalition and the influence that the governing coalition, as a bloc, has in the legislature. Since, in this paper, we are only concerned with the formation of majority coalitions under the simple majority rule, once formed, the governing coalition will have absolute influence over the legislative outcomes. Thus the influence the leading party has within the legislature is the same as its ability to influence the way the bloc will cast its vote on each issue in the legislature. If the 'internal' decision rule for the bloc is the simple majority rule, having a majority within the bloc will confer absolute influence upon

(1990), especially Chapter 3.

${ }^{6}$ For a thorough discussion of $P$-power or power as prize, see Chapter 6, Felsenthal and Machover (1998).

${ }^{7}$ See, for instance, Coleman (1971) for a statement to this effect. 
the leading party. ${ }^{8}$

With the preceding in mind and as we proceed with our continuing investigation into the role of voting power considerations in the political coalitionformation process, it appears reasonable for us to postulate an alternative hypothesis: that the party charged with forming the government will seek to enter into an arrangement that will maximize its absolute influence or $(I-)$ power in the legislature. And, the measure of $I$-power that is most relevant to our purpose is naturally the Penrose measure of absolute voting power, not the game-theoretic Shapley value as suggested by Aumann.

At this juncture, it is important to emphasize that, from our vantage point and consistent with casual observations, when the legislative vote goes in the direction of the ruling majority coalition, it does not preclude the possibility that the legislative outcome could also be favorable and beneficial to parties not within the ruling coalition. This clearly differentiates our vision of the coalition formation process from the game-theoretic view implicit in $\mathrm{Au}$ mann's approach in which those that are not members of the ruling coalition will end up with a zero expected payoff.

Using our approach, the empirical findings are considerably more positive and suggest to us that the Penrose measure may play a useful role in furthering our understanding of the coalition formation process. ${ }^{9}$ We report the details of our investigation below beginning with an outline of the Penrose measure and an example of how we have proceeded in computing the absolute influence of the leading party in the legislature. A description of the main hypothesis and its three variants follows. We then provide a brief description of the data sets employed and a discussion of our empirical findings. Some closing comments conclude the paper.

\section{The Penrose Measure of Absolute Voting Power or Influence}

As noted in Felsenthal and Machover (2004), the intuition underlying Penrose's measure may be summarized by two simple yet fundamental ideas. The first, simply put, says 'the more powerful a voter is, the more often will the outcome go the way s/he votes. Thus it is natural that the (a priori) power of a

\footnotetext{
${ }^{8}$ This last point is another important departure from Aumann's approach. In Aumann's approach, the Shapley-Shubik value of the leading party in the legislature is computed using a quota that is set equal to a simple majority of the entire legislature as opposed to a simple majority of the size of the alliance.

${ }^{9}$ At this juncture, one could argue that although the Penrose measure and the Shapley-Shubik index are not co-monotonic, departures are rare and therefore the Shapley-Shubik index will do just as well under the alternative definition of the quota used in the present investigation. This is indeed the case as our own earlier investigation has revealed. But it misses the point of our approach which emphasizes power as influence together with an associated calculus as opposed to power as prize in the game-theoretic sense with a different associated calculus.
} 
voter $v$ should be directly related to the proportion of all possible divisions of the legislature in which $\mathrm{s} / \mathrm{he}$ is successful. Let this proportion be denoted $r_{v}$. The second emphasizes that even a dummy will, through sheer luck, find itself on the winning side in half of all divisions. Isolating the pure luck element from the true influence yields a measure of the voter's influence that takes the following form as proposed in Penrose (1952): $\psi_{v}=2 r_{v}-1$.

$\psi_{v}[\mathcal{W}]$ is the Penrose $(I-)$ power of voter $v$ in some given simple voting game $\mathcal{W}$, and as pointed out in Felsenthal and Machover (2004), it also indicates the probability that voter $v$ is decisive. It is easy to verify that under the Penrose measure, a dummy will be assigned a value of zero whereas a dictator will be assigned a value equal to unity. Furthermore, if party $v$ is a member of an alliance $S$, then its overall Penrose $I$-power in the legislature is given by its Penrose measure within the alliance, reflecting its a priori ability to influence how its fellow alliance members vote in the legislature, multiplied by the alliance's Penrose $I$-power in the entire assembly. ${ }^{10}$

In simple terms, the coalition leader derives power or influence through a two-stage process: first, given the alliance's internal decision rule $\mathcal{W}_{s}$, the greater the coalition leader's ability to influence the collective position of the alliance, the greater is its internal influence. Second, the more influential the alliance is in the legislature, the greater will be the external influence the coalition leader will have in the legislature through the joint action of the alliance members.

In using the Penrose measure in our investigation, we have made specific assertions relating to the decision process within the legislature and within the majority coalition. Specifically, to secure the passage of a bill within the legislature, a simple majority of the total number of votes in the legislature suffices. As noted in our introductory remarks, our concern in this investigation is with the formation of majority coalitions. It is thus clear that no party outside the majority coalition wields any influence as long as the coalition remains a cohesive bloc that controls a majority of seats in the legislature. Only parties in the majority coalition wield power or influence and, as a bloc, the bloc wields absolute influence. In our investigation, we have also chosen the simple majority rule with a quota defined by the size of the majority coalition as the internal decision rule. We illustrate the computation of the measure below with an example before proceeding to a description of our hypotheses.

\section{Example 1 The 1920 Danish Assembly}

Consider the following 4-party situation denoted by [71; 42, 17, 49, 28]. In this

\footnotetext{
${ }^{10}$ As Felsenthal and Machover $(2002,2008)$ have explained, denoting the power of party $v$ within the alliance $S$ by $\psi_{v}\left(\mathcal{W}_{s}\right)$ and the power of the alliance in the assembly as $\psi_{\& s}\left(\mathcal{W} \mid \&_{s}\right)$, then the overall power of party $v$ which is a member of the alliance and is denoted $\psi_{v}\left(\mathcal{W} \| \mathcal{W}_{s}\right)$ is equal to $\psi_{v}\left(\mathcal{W}_{s}\right) \cdot \psi_{\& s}\left(\mathcal{W} \mid \&_{s}\right)$.
} 
example, the required majority to secure the passage of a bill is $71{ }^{11}$ The four parties in the legislature, ordered according to their position on the left-right ideological continuum, are respectively the Social Democrats with 42 votes, the Danish Social Liberal Party with 17 votes, the Liberals with 49 votes, and the Conservative People's Party with 28 votes - and the party asked to form the government is the Liberals, the party with the largest number of votes. An alliance with the Social Democrats will give the Liberal-led coalition a combined weight of 91 and hence a majority in the legislature. If this alliance were to form, then under the procedure that we have adopted, the Penrose measure for the leader, the Liberals, would be 1 . In contrast, if a majority alliance comprising the Social Democrats, the Danish Social Liberal Party and the Liberals were to form with a combined weight of 108 votes, the Penrose measure of the leader would be $1 / 2$ since, with the inclusion of the Danish Social Liberal Party, the Liberal party would lose its decisive absolute majority within the majority coalition. ${ }^{12}$

\section{The Main Hypothesis and Variations}

\subsection{The Main Hypothesis}

When charged with forming a government, we hypothesize that the party so appointed will act in a manner that will maximize its chance of implementing its policy agenda and accordingly will act in a manner consistent with the maximization of its Penrose ( $I$-) power, a measure that is now widely re-

\footnotetext{
${ }^{11}$ The data are taken from de Swaan (1973:269). A simple majority of 71 implies that there were at that time 140 members in the Danish parliament. However, the total number of seats of the four listed parties is only 136. This gap is explained by the fact that de Swaan ignored small parties (or independents) who controlled no more than $2.5 \%$ of the seats in parliament because such parties were very seldom included in governmental coalitions.

${ }^{12}$ The Penrose measure of a priori voting power of a voter $v$, denoted $\psi_{v}$, is equal to the number of winning coalitions in which $v$ is critical divided by the number of coalitions to which $v$ belongs. A coalition is winning if it has sufficient votes to pass a decision, otherwise it is losing. A voter is critical if his defection from a winning coalition renders it losing, or if his joining a losing coalition renders it winning. For $n$ voters there are altogether $2^{n}$ coalitions (or bi-partitions) of which every voter belongs to $2^{n-1}$ coalitions. In the above example let us consider the alliance consisting of three members whose weights are 42,17 , and 49 . This is a winning alliance (coalition) because its combined votes (weights) is 108 - which exceeds the quota of 71 . If we assume that the internal decision rule of this alliance is a simple majority of its members' weights (55), then there are altogether four (internal) coalitions within this alliance in which the member with weight 49 (the coalition leader) exists - $\{49\},\{49,17\},\{49,42\},\{49,42,17\}$ - of which this member is critical in the second and third coalitions. Consequently the coalition leader's internal (direct) voting power in this case is $2 / 4=0.5$. But since the alliance as a whole controls an absolute majority of the votes within the parliament - and hence is a dictator whose a priori voting power is 1 - it follows that the overall (indirect) a priori voting power of the coalition leader in this case is equal to its internal voting power within the alliance multiplied by the alliance's power within the parliament, i.e., $0.5 \cdot 10.5$.
} 
garded as a reasonable index for measuring the a priori absolute influence of a party in an $n$-party decision-making situation with a given quota. For convenience, we shall refer to this hypothesis as the Maximal PI hypothesis. Applying this hypothesis to the situation described in Example 1, it is clear that the Liberals, the party charged with forming the government, will prefer the first arrangement in which its measured a priori Penrose power is unity to the second in which the corresponding Penrose value is only $1 / 2$ and if there are no other arrangements that will confer 'dictatorial' power on the Liberals, then the hypothesis would predict that the coalition comprising the Social Democrats and the Liberals will form. This, however, is not the case. There are two other arrangements that will also confer 'dictatorial' power on the coalition leader, specifically, the coalition comprising: (a) the Danish Social Liberal Party, the Liberals and the Conservative People's Party; and (b) the Liberals and the Conservative People's Party. This observation immediately brings to the fore a basic difficulty with our hypothesis in its present form; it lacks parsimony, a weakness that it shares with Aumann's hypothesis. A second example drawn from the 1936 Swedish election data reinforces this point.

\section{Example 2 The 1936 Swedish Assembly}

Consider the 5-player weighted voting game given by [116; 6, 112, 36, 27, 44]. In this game any coalition which controls at least 116 votes is winning and the parties, ordered according to their position on the left-right ideological continuum, are respectively the Communist Party of Sweden (6 votes) , the Social Democrats (112 votes), the Farmers' League (36 votes), the Liberal Party (27 votes) and the Right-wing Conservative Party (44 votes). ${ }^{13}$ In this instance, the party charged with forming the government is the Social Democrats which controlled 112 votes in the legislature. There are altogether 15 majority coalitions that the Social Democrats could consider when forming a majority government. In all except the grand coalition, however, the coalition leader has an absolute majority and thus in 14 out of the 15 instances, its Penrose $I$-power is unity. The predicted set in this instance thus comprises 14 out of the 15 possible majority coalitions, indicating clearly a lack of parsimony in the theory and rendering the prediction from the theory of little value.

\footnotetext{
${ }^{13}$ The data are taken from de Swaan (1973: 260) who ignored in this case small parties controlling together five seats.
} 


\subsection{Three Variations}

From the perspective of developing a predictive theory of coalition formation, it is important that one has a theory that provides a reasonably precise prediction as to the likely outcome. Parsimony - in terms of the size of the predicted set of likely coalitions - is an important attribute of a good predictive theory and, therefore, to avoid the profusion of possibilities under the maximal PI hypothesis, we considered three variations of the main hypothesis while maximizing, minimizing, or holding constant one or more additional variables.

\subsubsection{Variation I: Restriction to Closed Coalitions}

In this variation, we consider a restriction of the domain to the set of closed or ideologically connected coalitions. By a 'closed' coalition, we mean that there are no ideological 'gaps': if two parties belong to the coalition then any party that lies ideologically between them belongs also to the coalition; otherwise the coalition is 'open'. Returning to Example 1, of the three majority coalitions involving the coalition leader and in which the leader has the maximal Penrose value of 1 , only two are closed.

The motivation for introducing this restriction is intuitive. In a multiparty system, parties that are diametrically-opposed in ideology do not usually enter into a political alliance except when the situation demands it, such as in matters that involve the national security. Under ordinary circumstances, an alliance of this nature is likely to increase the cost of the arrangement to the leading party and without doubt significantly increase the vulnerability of the alliance to defection. Thus it is unlikely that a party that is charged with forming a governing coalition under normal conditions will enter into such an arrangement even though such a union may result in the highest value for the leader's Penrose I-power. Corroborative evidence indicating a preference by political parties for closed coalitions is provided in de Swaan (1973:148); out of 108 majority coalitions in the nine countries which he investigated, 85 (or 79 percent) of these were closed coalitions.

At the operational level, the restriction to closed coalitions will help to increase the parsimony of the hypothesis which, under the given restriction, we shall refer to as the Closed Maximal PI hypothesis. ${ }^{14}$

\footnotetext{
${ }^{14}$ It is important to make the distinction between: (a) restricting the domain to closed coalitions and picking an arrangement that is maximal from the restricted set; and (b) picking an arrangement from the maximal set that satisfies the restriction. Although (a) and (b) may pick the same arrangement, it is the former that is employed in our analysis in order to test the hypothesis that the maximization of the leader's voting power in forming a governmental coalition is an important consideration only (or mainly) if the coalition is closed.
} 


\subsubsection{Variation II: Closed Maximal PI Coalitions of Minimum Size}

Even with the domain restricted to the set of closed coalitions, the size of the predicted set may still be large. We thus consider a further restriction of the domain to only those closed and maximal arrangements that are also of minimum size. This will yield predicted sets that are subsets of those obtained under Variation I and, like the Gamson-Riker minimum size principle, ${ }^{15}$ will yield predicted sets that are often singletons or that involve a relatively small number of coalitions. We shall refer to this variation of the hypothesis as the Closed Maximal PI Minimal Size hypothesis. ${ }^{16}$

\subsubsection{Variation III: Closed Maximal PI Coalitions of Minimal Range}

As an alternative to the restriction of the predicted set to only those closed maximal coalitions that are of minimal size, we also consider the restriction of the predicted set to the subset of closed maximal coalitions that are of minimal range. As noted previously, on a policy scale in which any two adjacent parties are regarded as one unit of distance apart, the range of a coalition is the distance between the two parties in the coalition whose positions on the policy scale are furthest apart. Inclusion of this variation is largely motivated by de Swaan's finding that Leiserson's (1966) and Axelrod's (1970) closed minimal range hypothesis appears to fit the historical data rather well. Of the 85 closed coalitions that were observed in de Swaan's study, 55 were of minimal range (see de Swann, 1973: 148). It also adds a dimension to the optimization process emphasizing, in addition to power, the desirability for homogeneity in the ideological positions of coalition members, an idea that is also rather intuitive. We shall refer to this other variant as the Closed Maximal PI Minimal Range hypothesis.

Summarizing, in addition to considering the maximal PI hypothesis, we shall also consider in our investigation: (a) the variation that restricts the maximization process to only those majority coalitions that are ideologically closed; (b) the variation that applies a further restriction to the predicted set to include only those with minimal size; and (c) the variation that further restricts the prediction of the Closed Maximal PI version to the subset that is of minimal range. While (b) may be regarded as an analogue to the Gamson-Riker minimum size principle, (c) may be viewed as the analogue to the Leiserson-Axelrod closed minimal range theory.

\footnotetext{
${ }^{15} \mathrm{~A}$ minimum size coalition is a winning coalition that controls no more seats in the legislature than any other winning coalition. Both Gamson (1961) and Riker (1962) predicted that the (winning) coalition that will actually form in coalition games is likely to be of minimum size.

${ }^{16} \mathrm{Such}$ an approach is however distinct from the minimum size principle and it also overcomes the objection raised by Aumann concerning coalitional stability when the minimum size principle is invoked.
} 


\section{Data, Analysis and Findings}

\subsection{Data Sources}

In testing our main hypothesis and its different variants, we made extensive use of the tabulation of election outcomes provided in de Swaan's (1973) book Coalition Theories and Cabinet Formations. This data tabulation covers nine countries and selected historical parliamentary elections: Denmark (1918-1971), Sweden (1917-1970), Norway (1933-1936; 1965-1969), Israel (1949-1969), Italy (1946-1972), The Netherlands (1918-1972), Finland (1919-1972), Germany's Weimar Republic (1919-1932), and France's Fourth Republic (1945-1957). For our purpose, de Swaan's data is particularly useful because, for each election, in addition to listing the number of seats controlled by parties which gained at least $2.5 \%$ of the seats in an assembly, the parties have also been ordered along the left-right ideological continuum. These rank-orderings of parties render the task of identifying closed majority coalitions in each election considerably less onerous.

Differing somewhat from de Swaan's study, however, our concern is with the formation of original coalitions, that is, coalitions that are formed immediately following a general election. Interim coalitions are not included in our analysis because their formation may be due to reasons which clearly cannot be attributed to the leading party's desire to maximize its a priori voting power, e.g., the death of the former prime minister, the disintegration of one of the coalition's parties, or the defection of some members from one party within the coalition to another. In implementing our empirical analysis, we also considered only those winning coalitions that included the coalition leader which we identify ex post as the party of the prime minister. These considerations have meant that only 65 original coalitions across the nine countries are included in our analysis.

In part, because de Swaan (1973:237) has identified Israel as 'a difficult country for the theories' and, in part, because, in the case of Israel, very small parties were sometimes included in governmental coalitions, we have performed additional analysis covering all 18 Israeli elections from 1949 through 2006. In this analysis, we included all parties that gained representation in Israel's parliament. This is in contrast to de Swaan's analysis of Israel that included only elections up to 1969 and covering only those parties that controlled more than $2.5 \%$ of the seats in the various parliaments.

\subsection{Evaluating the Worth of a Theory}

No theory is expected to correctly predict the outcome all of the time. In some contexts, a theory that performs marginally better than chance may be considered a reasonably good theory if other competing theories can do 
no better. Intuitively, when a restriction is placed on the set of admissible coalitions resulting in predicted sets that are more precise, it would appear that the frequency of obtaining a correct prediction is likely to be lower compared with the case when the domain is unrestricted. This is certainly the case when the restriction results in predicted sets that are proper subsets of the unrestricted predicted sets. This does not however automatically render the restricted theory a poorer predictive theory. Conversely, for the same election, different theories may give rise to predicted sets that differ considerably in terms of size, and the theory that gives rise to a larger predicted set will naturally have a better chance of correctly predicting the outcome. But such a theory is not necessarily a better predictive theory. Somehow, the tradeoff between the probability that the actual outcome is included in the predicted set and the parsimony of the predicted set will have to enter the calculus in determining which theory should be preferred.

In determining the worth of each theory in our analysis, we have kept in mind this tradeoff. Instead of attempting a direct comparison of the competing theories, our approach is to compare the different theories with their respective randomized counterparts so that the evaluation of each theory is carried out on a level playing field. For the de Swaan data set, given a reasonably large sample size of 65 , we carry out this evaluation by invoking the Central Limit Theorem. In the case of our detailed investigation of Israel in which only 18 elections are involved, such a procedure would be inappropriate. Here, we compute the exact probability mass function under each theory to facilitate our assessment of the theories. These two evaluation procedures are outlined below.

Given the distribution of seats controlled by the various political parties in the $i$-th election, $(i=1,2, \ldots, n)$, let $N_{i}$ denote the number of possible winning coalitions ${ }^{17}$ that include the leading party, that is, the party charged with forming the government. If the leading party is successful in forming the government, the outcome will necessarily be one of these winning coalitions. Let $S_{i j}$ denote the number of winning coalitions in the predicted set under the $j$-th theory $(j=1,2, \ldots, m)$. If $S_{i j}=1$, then the theory in question effectively makes a unique prediction as to the government that will form in the $i$-th election. If the actual outcome coincides with the predicted outcome, we consider the theory as 'successful' or having produced a consistent prediction; otherwise we consider the theory as having 'failed. ${ }^{18}$ If the prediction of the theory in question is no better than that of a pure chance mechanism, then the probability of a consistent prediction would be $1 / N_{i}$,

\footnotetext{
${ }^{17}$ In this paper, a winning coalition is defined as one that controls over half of the seats in the legislature.

${ }^{18}$ In a more general context, a prediction is regarded as consistent as long as the actual outcome is included in the predicted set.
} 
implying that each of the $N_{i}$ winning coalitions is equally likely. More generally, if $N_{i}>S_{i j}>1$, this probability, which we now denote by $P_{i j}$, would equal $S_{i j} / N_{i}$. Needless to say, when $S_{i j}=N_{i}$, the predicted set coincides with the set of all winning coalitions that include the leading party and $P_{i j}=1$. In this instance, the actual outcome is definitely contained in the predicted set. But a theory that does this would neither be interesting nor useful.

As the distribution of seats among political parties differs across the $n$ elections, the size of the set of winning coalitions for each of these $n$ elections $\left(N_{1}, N_{2}, \ldots, N_{n}\right)$ and the corresponding sizes of the predicted set under the $j$-th theory $\left(S_{1 j}, S_{2 j}, \ldots, S_{n j}\right)$ cannot be expected to remain constant. Consequently, if the prediction of the $j$-th theory were no better than a chance mechanism, the probability $\left(P_{i j}\right)$ that the prediction of the $j$-th theory is consistent with the actual outcome will also vary across the $n$ elections. As long as the outcomes are independent across elections, the expected number of consistent predictions under the $j$-th theory will be given by $\sum_{i} P_{i j}$ with associated standard error $\left[\sum_{i} P_{i j}\left(1-P_{i j}\right)\right]^{1 / 2}$. We refer to the quantity $\sum_{i} P_{i j}$ as the randomized mean for the $j$-th theory.

For sufficiently large $n$, the Central Limit Theorem for independent random variables postulates that the expected number of consistent predictions will be approximately normally distributed with mean $\sum_{i} P_{i j}$ and standard error $\left[\sum_{i} P_{i j}\left(1-P_{i j}\right)\right]^{1 / 2} .{ }^{19}$ In our analysis of de Swaan's data, we exploit this result when evaluating the worth of the $j$-th theory by comparing the actual number of consistent predictions obtained under the $j$-th theory with that obtained under its randomized counterpart. More precisely, we measure the standardized deviation of the actual number of consistent predictions of the $j$-th theory from its randomized mean.

Our supplemental analysis of the Israeli elections, however, requires a slightly different approach. First, the data set comprises only 18 elections, a sample size that is too small to invoke the Central Limit Theorem. Additionally, the probability of a consistent prediction $\left(P_{i j}\right)$ in each of these 18 elections is close to zero making the actual probability mass function severely skewed. While there are a number of alternative approaches that one may consider when evaluating the worth of each theory, we opted in this paper

\footnotetext{
${ }^{19}$ The Central Limit Theorem for independent variables states the following. Let $X_{1}, X_{2}, \ldots X_{n}$ be a sequence of independent random variables having respective means and variances $\mu_{i}=$ $E\left(X_{i}\right), \sigma_{i}^{2}=\operatorname{Var}\left(X_{i}\right)$. If (i) the $X_{i}$ are uniformly bounded; that is, for some $M,\left\{P\left|X_{i}\right|<\right.$ $M\}=1$ for all $i$, and (ii) $\sum_{i=1}^{\infty} \sigma_{i}^{2}=\infty$, then

$$
P\left\{\frac{\sum_{i=1}^{n}\left(X_{i}-\mu_{i}\right)}{\sqrt{\sum_{i=1}^{n} \sigma_{i}^{2}}} \leq a\right\} \rightarrow \frac{1}{\sqrt{2 \pi}} \int_{-\infty}^{a} e^{-x^{2} / 2} d x \text { as } n \rightarrow \infty .
$$
}

See Ross (2002). 
to compute the exact probability mass function for the randomized scheme under each of the theories, noting that the probability mass at $k$, the number of consistent predictions under the $j$-th theory, is given by:

$$
P_{j}(x=k)=\sum_{a_{k} \in A_{k}}\left(\prod_{i \in a_{k 1}} P_{i j} \prod_{i \notin a_{k 0}}\left(1-P_{i j}\right)\right)
$$

In this expression, $A_{k}$ denotes the set of situations that gives rise to $k$ consistent predictions and $a_{k}$ is an element of this set. The cardinality of the set $A_{k}$ is ${ }^{n} C_{k}$ (i.e. the number of combinations of size $k$ one can extract out of the set of $n$ elections) and the summation in the expression is over each of these ${ }^{n} C_{k}$ different situations. In each of these situations, the set $a_{k 1}$ which has cardinality $k$ refers to the set of elections in which the theory has produced a consistent prediction. The set $a_{k 0}$ with cardinality $(n-k)$, on the other hand, refers to those elections in which the actual outcome is not in the predicted set.

As an illustration, consider a sequence of three elections, that is, $n=3$. For the $j$-th theory, suppose $P_{1 j}, P_{2 j}$ and $P_{3 j}$ are respectively $1 / 2,1 / 3$ and $1 / 4$. If this theory is no better than a chance mechanism, there will be ${ }^{3} C_{0}$ or exactly one instance in which it will fail to produce a consistent prediction. This is the case when $k=0$ and denoting a consistent prediction by the letter $s$ and an inconsistent prediction by the letter $f$, the sequence of outcomes referred to in this instance is fff. The probability that this occurs is given by $P_{j}(x=0)\left(1-P_{1 j}\right)\left(1-P_{2 j}\right)\left(1-P_{3 j}\right)=1 / 2 \cdot 2 / 3 \cdot 3 / 4=1 / 4$. Similarly, there will be ${ }^{3} C_{1}$ or exactly three instances in which the theory will produce exactly one consistent prediction. These three instances are associated with the outcome sequences $s f f, f s f$, and $f f s$. Thus the probability that the theory achieves exactly one consistent prediction, $P_{j}(x=1)=P_{1 j}\left(1-P_{2 j}\right)\left(1-P_{3 j}\right)+\left(1-P_{1 j}\right) P_{2 j}(1-$ $\left.P_{3 j}\right)+\left(1-P_{1 j}\right)\left(1-P_{2 j}\right) P_{3 j}$ and this equals $1 / 4+1 / 8+1 / 12=11 / 24$. It can be similarly verified that $P_{j}(x=2)=1 / 4$ and $P_{j}(x=3)=1 / 24$. As always, these probabilities sum to unity as the different scenarios are both mutually exclusive and exhaustive.

With the exact probability mass function for each theory in hand and supposing the number of consistent predictions under the $j$-th theory is $k_{j}^{*}$, we are able to calculate the exact probability that this or a larger number of consistent predictions will be observed under its randomized counterpart scheme, i.e. we can calculate $P_{j}\left(x \geq k_{j}^{*}\right)$. This, in turn, will enable us to test the null hypothesis that the theory in question is no better than a pure chance mechanism. A large value for $P_{j}\left(x \geq k_{j}^{*}\right)$ is indicative that this is indeed the case, whereas a very small value for $P_{j}\left(x \geq k_{j}^{*}\right)$ provides strong evidence against the null hypothesis, indicating that the theory in question strongly outperforms its randomized counterpart in its predictions and thus 
is a candidate deserving further consideration.

\subsection{Empirical Findings}

Detailed computational results of our empirical work based on de Swaan's data set appear in Tables A.1 and A.2 in the Appendix. In Tables A.3 and A.4 in the Appendix, we list the details from our analysis of the separate set of 18 Israeli elections and the exact probability mass functions that we employed. In all these tables there are entries of the type $x / y$ in the columns of the various tested theories. Thus, for example, in the first row of Table A.1 under the maximal PI column appears the entry 16/98. This entry should be interpreted thus: of the total 98 possible coalitions in which the leader's Penrose measure is maximized over all 17 elections considered, 16 coalitions were actually formed. The same interpretation applies, mutatis mutandis, to all other entries of this form. The key findings from our analyses are summarized in Table 1 below.

The Maximal PI hypothesis performs exceedingly well securing the largest number of predictive successes. The number of consistent predictions is highest of all theories considered: 43 consistent predictions out of 65 elections for de Swaan's data set (cf. Table A.1) and 11 consistent predictions out of 18 elections for the Israeli 1949-2006 data set (cf. Table A.3). But as is readily verified by examining the columns labeled Maximal PI in Tables A. 2 and Table A.3, in many elections the size of the predicted set under this hypothesis is large relative to the number of winning coalitions. Take for instance the 1936 election to the Swedish Assembly: of a total of 15 winning coalitions, 14 (or 93 percent) are included in the predicted set under this hypothesis. Likewise, for the 1969 Israeli election reported in Table A.3: of 4069 possible winning coalitions, 3905 (or 96 percent) are contained in the predicted set. Not surprisingly, therefore, despite the large number of consistent predictions, its performance for the de Swaan data set is only 4.1831 standard errors above its randomized mean, the lowest obtained of all the hypotheses considered. Similarly, in the case of the more detailed Israeli elections data, despite having achieved 11 out of 18 predictive successes, its performance is among the worst of all hypotheses that managed to score at least one success.

Inferring from the exact probability mass function for its counterpart randomized scheme as detailed in Table A.4, the probability of observing 11 or more consistent predictions under the randomized scheme is 0.051 , indicating a 5.1 percent chance that this event will occur. Thus while at the 90 percent confidence level the conclusion that its performance is significantly different from its purely randomized counterpart cannot be rejected, at the 99 percent confidence level this position will be squarely rejected. In sum, under 


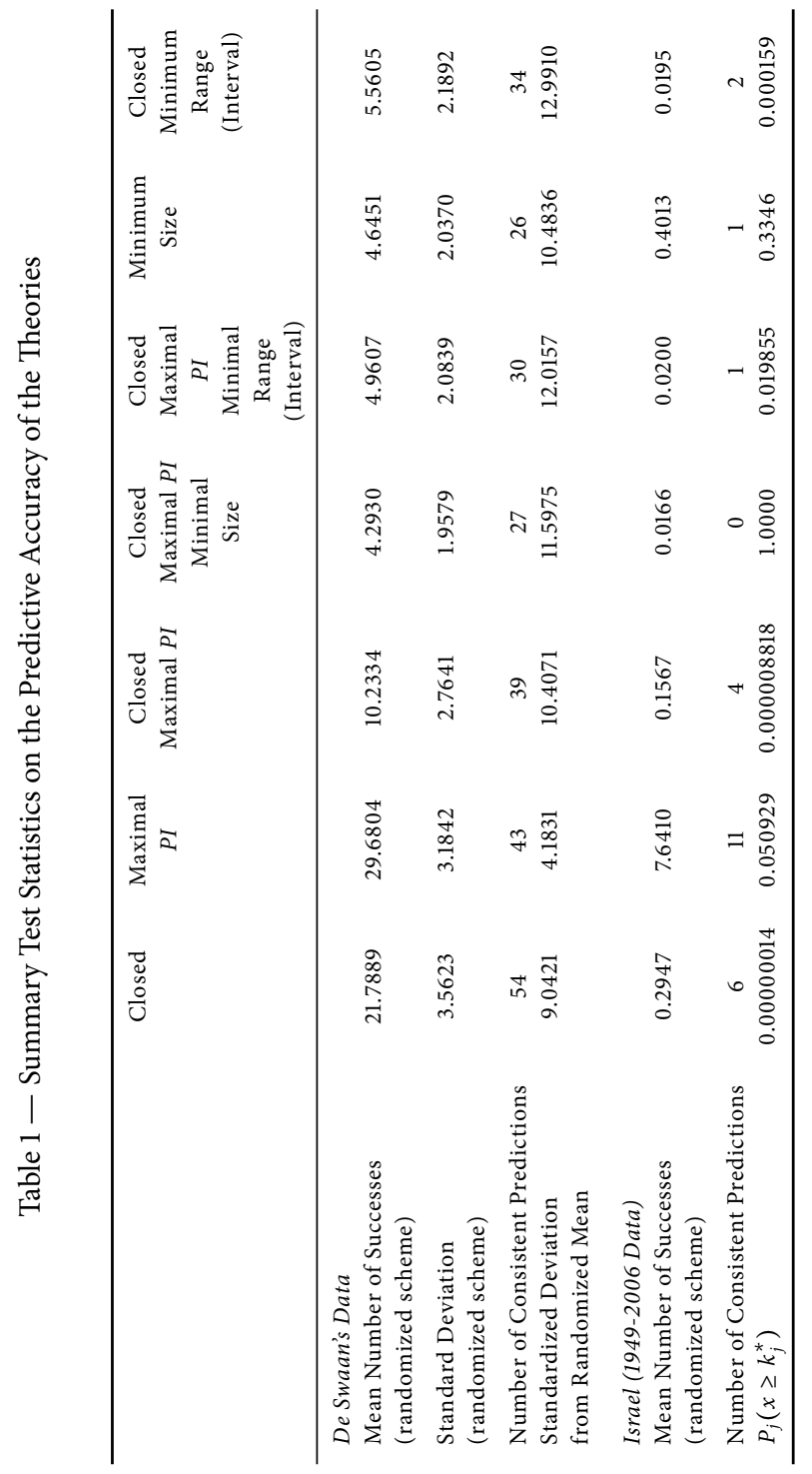


the given circumstances, the lack of parsimony of the maximal PI hypothesis makes it an unattractive candidate as a predictive theory of coalition formation.

For the purpose of comparison, we also investigated the predictive performance of the cheapest coalition or minimum size principle. Like the maximal $P I$ hypothesis, it is simple and the informational requirements are similar. But unlike the maximal PI hypothesis, it is extremely parsimonious and often produced predicted sets that are very small relative to the number of winning coalitions. This principle appears to work reasonably well for the de Swaan data set, managing 26 consistent predictions in 65 elections or 10.484 standard errors above its randomized mean. For the detailed analysis of the 18 Israeli elections, however, it predicted the outcome of only one election correctly. Additionally, when its performance is compared with those achieved under other variations of the maximal PI hypothesis, which we shall discuss shortly, this theory appears to be a dominated theory.

Unlike the hypotheses just discussed, all three variants of the maximal PI hypothesis considered in this investigation require that the selected coalitions be closed on the left-right continuum. This imposes the additional informational requirement that the political parties are ranked accordingly. But this additional burden on the investigator appears to be amply compensated for by the very decent performance of two of the variations, especially by the Closed Maximal PI hypothesis and the Closed Maximal PI Minimal Range hypothesis.

When the Closed Maximal PI hypothesis is confronted with de Swaan's data set, it holds up well, achieving 39 successes out of 65 and a performance of 10.407 standard deviations above its randomized mean. It also performed exceedingly well in our supplementary analysis of the 18 Israeli elections and is probably the best performing of all hypotheses considered for this second data set, achieving 4 consistent predictions out of 18 . We note that under the corresponding randomized scheme, the probability of observing 4 or more consistent predictions is only 0.000008 or practically zero. This is indicative that the level of predictive success achieved by the hypothesis are almost surely not the result of pure chance.

Equally encouraging results were obtained under the Closed Maximal PI Minimal Range hypothesis. This hypothesis which focuses attention only on those closed maximal PI coalitions that are of minimal range - worked well for the two data sets, producing predictions that are on par with those under the Leiserson-Axelrod closed minimal range theory, the latter being probably the best known predictive theory currently. The corresponding results for the Leiserson-Axelrod theory are reported in Table 1 for the purpose of comparison.

Under this variation of our alternative hypothesis, the level of predictive 
success obtained using the de Swaan data set is 30 out of 65 elections and this compares favorably with 34 out of 65 elections under the Leiserson-Axelrod closed minimal range theory; and in the case of the Israeli 1949-2006 data set, the relative performance of the two theories is one out of 18 and two out of 18 consistent predictions, respectively. It should be noted that except for one specific instance, this variation of the maximal PI hypothesis leads to predicted sets that are no larger than those under the Leiserson-Axelrod closed minimal range theory. In fact, in a number of instances, the predicted set under this variation is marginally smaller.

The variation that we have referred to as the Closed Maximal PI Minimum Size hypothesis, as expected, makes relatively precise predictions about the possible coalitions that will form in each situation but without the inherent instability property associated with the minimum size principle. This variation worked well under the de Swaan data set but did not produce a single consistent prediction in respect of the 18 Israeli elections thus leading us, as in the case of the minimum size principle, to reject the hypothesis.

\section{Concluding Remarks}

So, how useful are a priori power indices in predicting the formation of political coalitions? Do theories based on these indices work?

From our empirical investigation of Aumann's hypothesis (cf. Chua and Felsenthal, 2008), which emphasizes a central role for the Shapley-Shubik index in the theory of coalition formation, we obtained an outcome that was largely negative. The prognosis flowing from the Felsenthal and Machover (2008) re-analysis of 77 alliances investigated in our test of Aumann's hypothesis is, at first blush, also not particularly promising. In 49 of the 77 alliances re-examined, the coalition leader is a posteriori a dictator, indicating in each of these 49 instances that the other members of the governmental alliance are a posteriori dummies. Furthermore, in 21 additional alliances, at least one member lost power or became a dummy after joining the alliance, raising the possibility that voting-power considerations leading to the formation of what Felsenthal and Machover $(2002,2008)$ called 'feasible' or 'expedient' alliances play no significant role in the formation of actual governmental alliances. ${ }^{20}$

But, from the perspective of the coalition leader, these observations are perhaps not surprising and even indicative that a priori measures of power do, in fact, play a role in the formation of governmental coalitions. Quite

\footnotetext{
${ }^{20}$ Felsenthal and Machover $(2002,2008)$ defined a feasible alliance as one in which the overall absolute voting power of each member of the alliance is not smaller than his voting power when no alliance exists. An expedient alliance is defined as one in which the overall absolute voting power of each member of the alliance is larger than his voting power when no alliance exists.
} 
naturally, one would anticipate that a coalition leader would have an incentive to maneuver, as far as is possible, the coalitional arrangement to one in which $s /$ he is a dictator within the alliance in the technical sense of the word. It would indeed be surprising if the opposite were true, that is, the coalition leader is a posteriori a dummy. Where the use of power indices fails is in their ability to explicitly capture the compromise that the coalition leader made in securing the a posteriori dictatorial or near dictatorial role. This, perhaps, may be a reason why, in our present analysis, the Closed Maximal PI and the Closed Maximal PI Minimal Range hypothesis performed relatively well; because the additional restriction(s) may have implicitly, perhaps only partially, captured the extent of the compromise that the coalition leader is required to make.

Viewed from this perspective, the measure of success achieved by the Penrose measure as reported in the present paper suggests that further work should be carried out to more carefully investigate the role played by votingpower considerations in the formation of political coalitions.

\section{Appendix}

This appendix consists of four tables. See overleaf. 


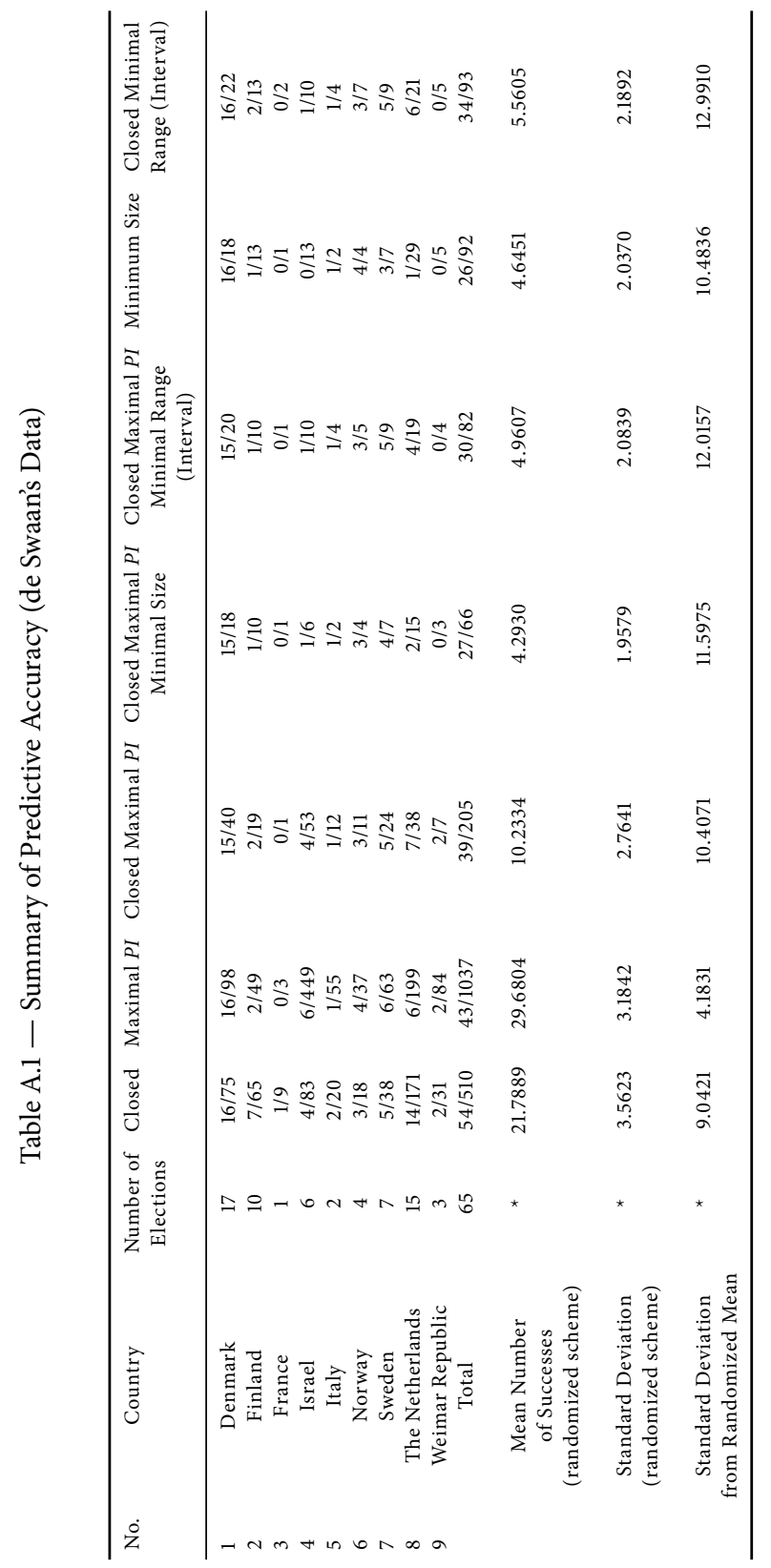




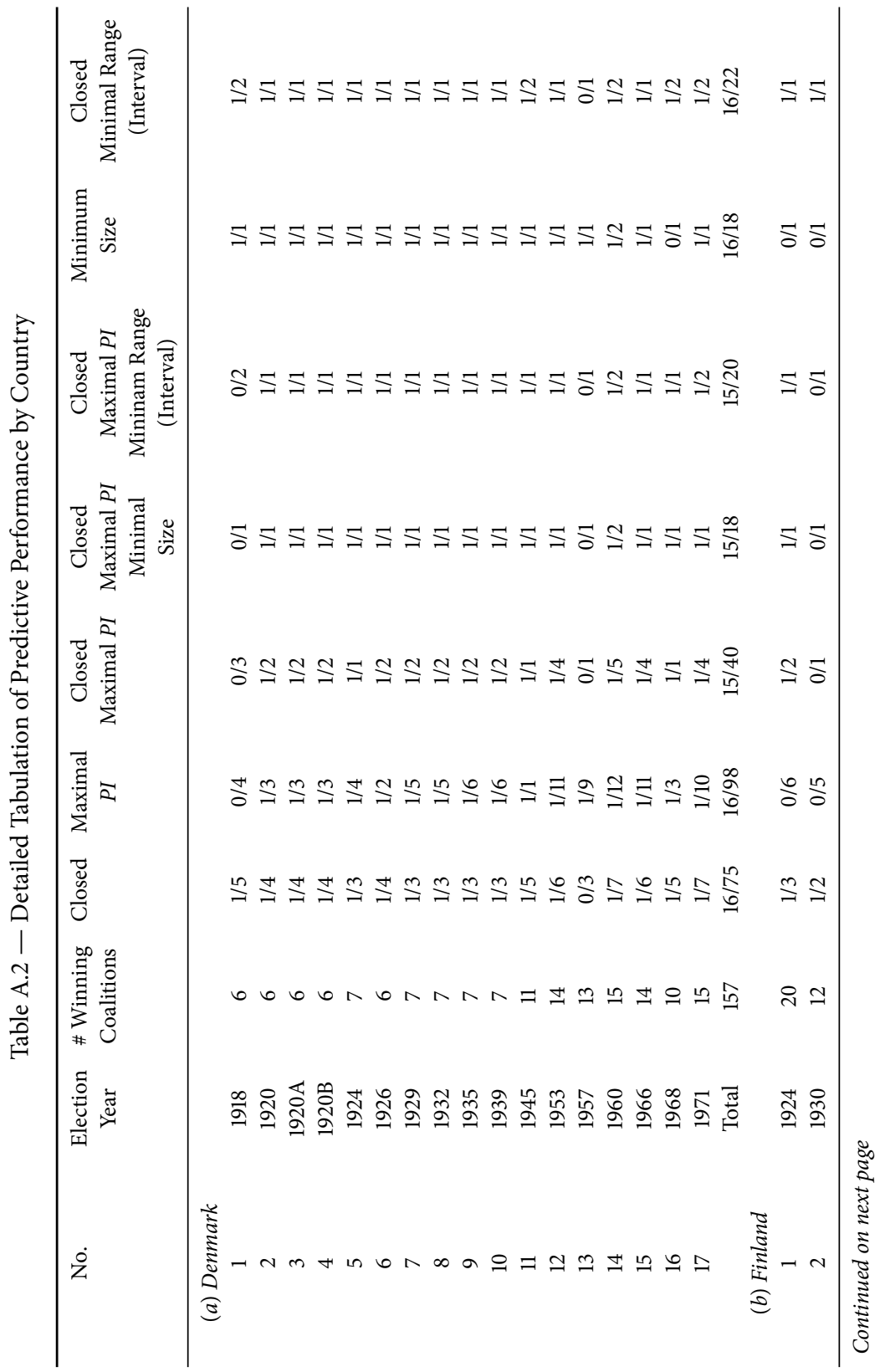




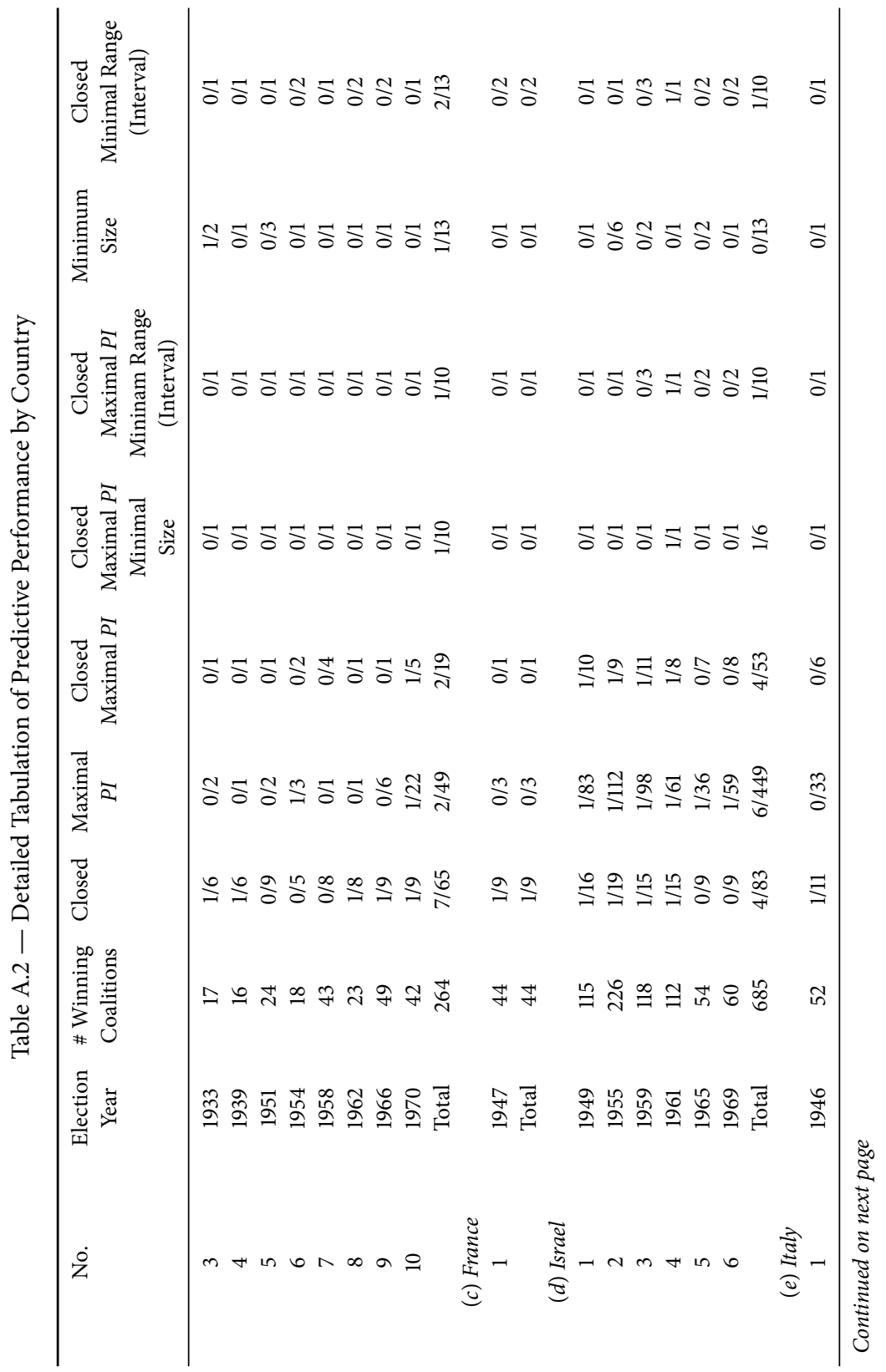




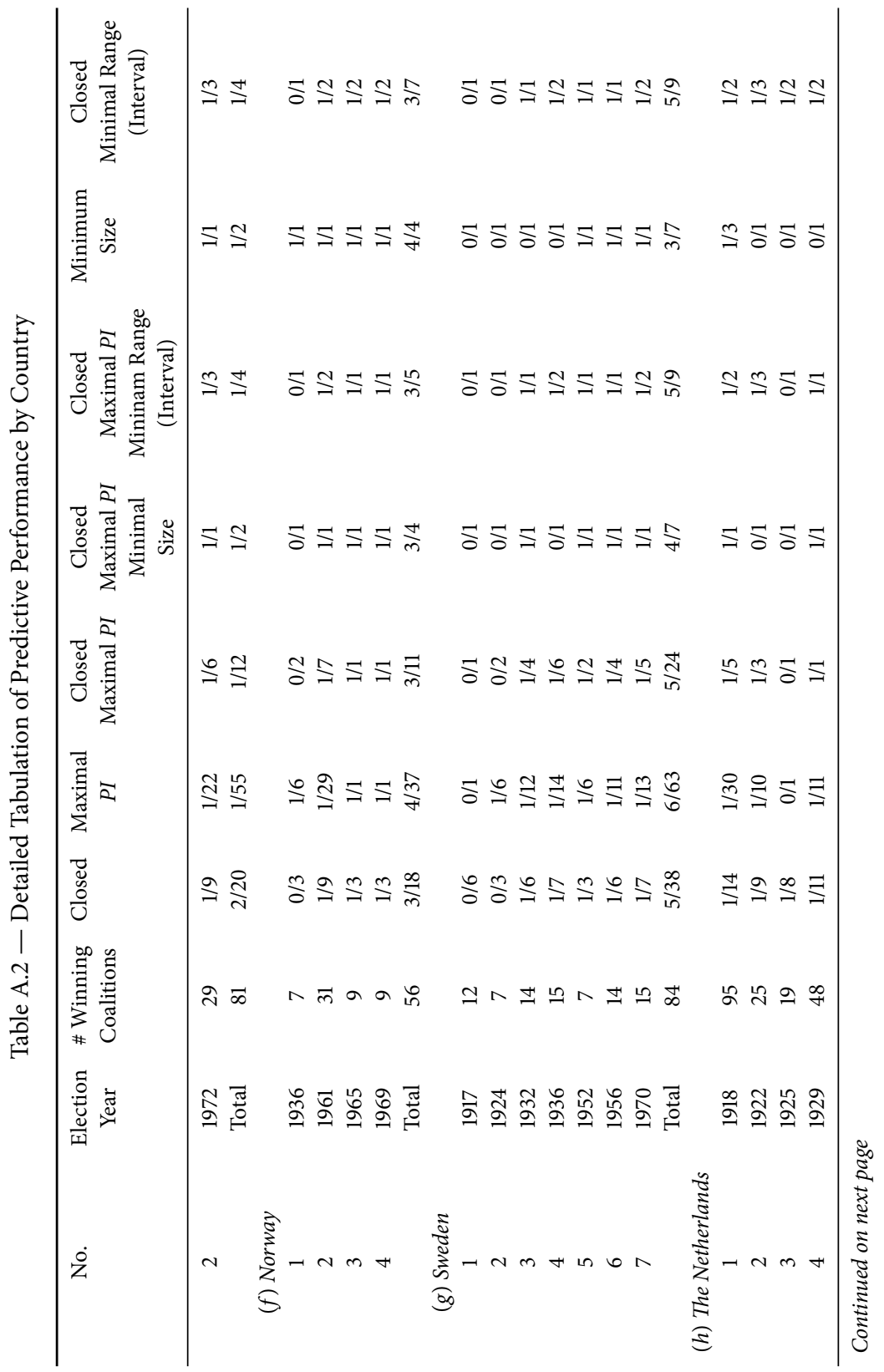




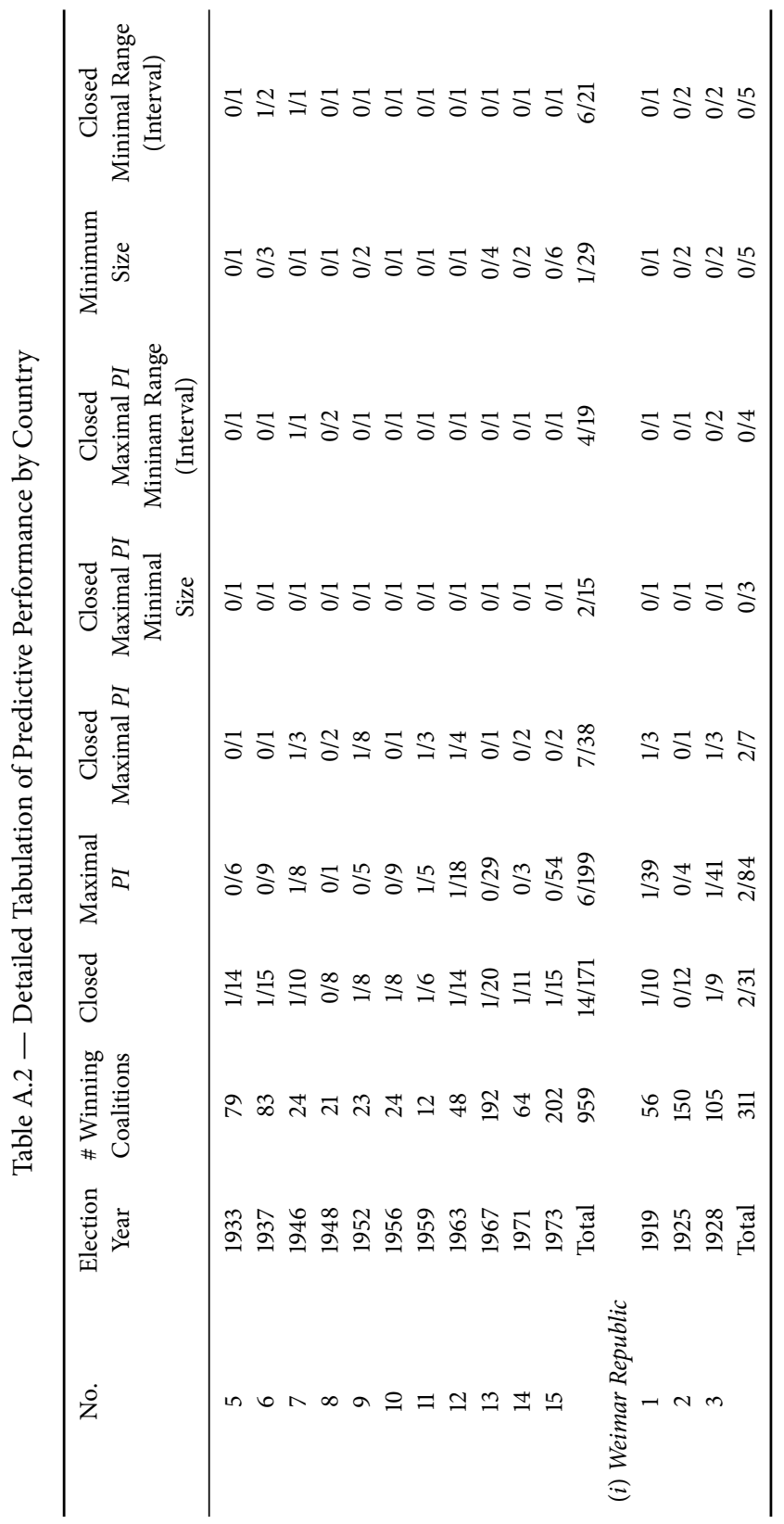




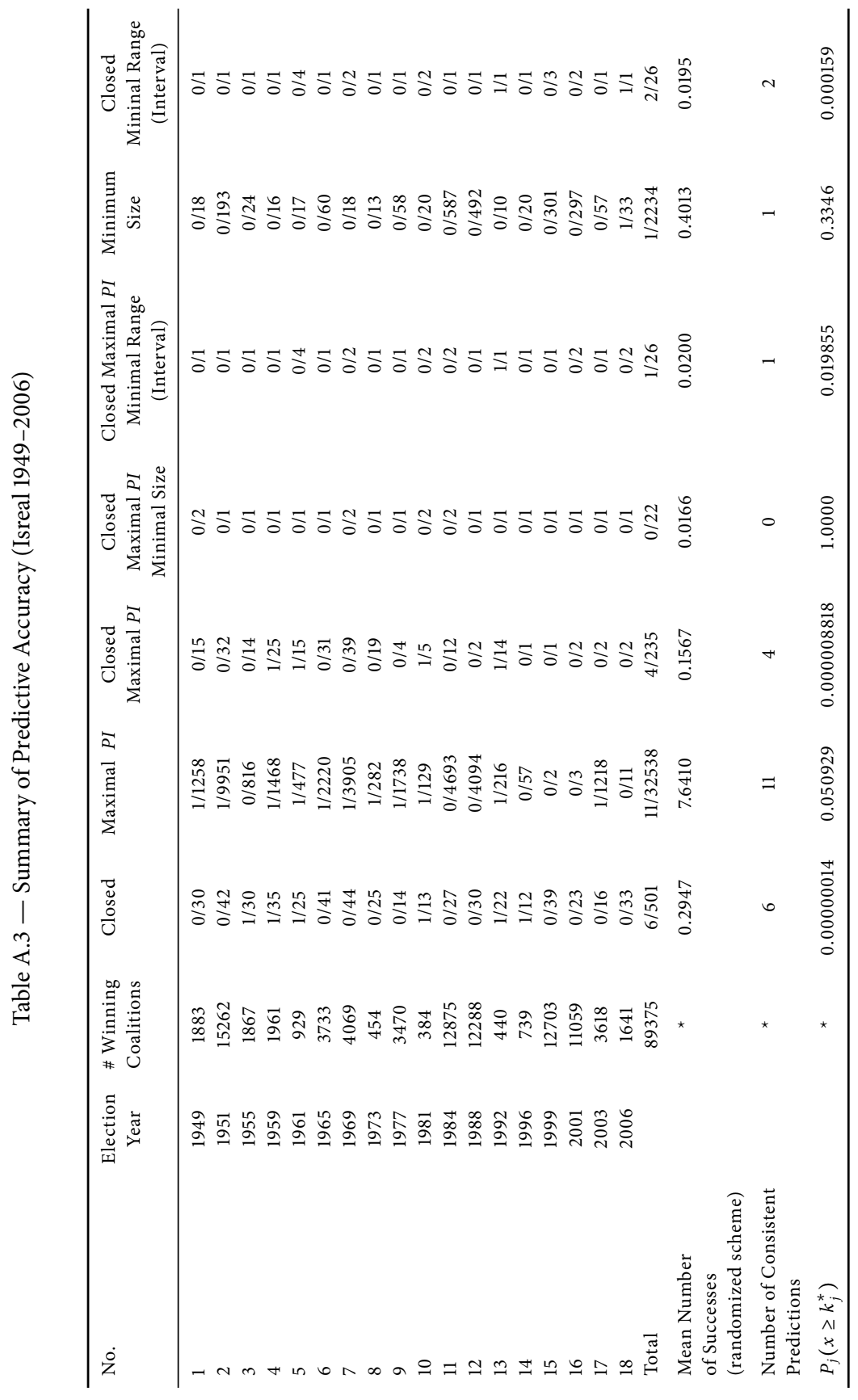




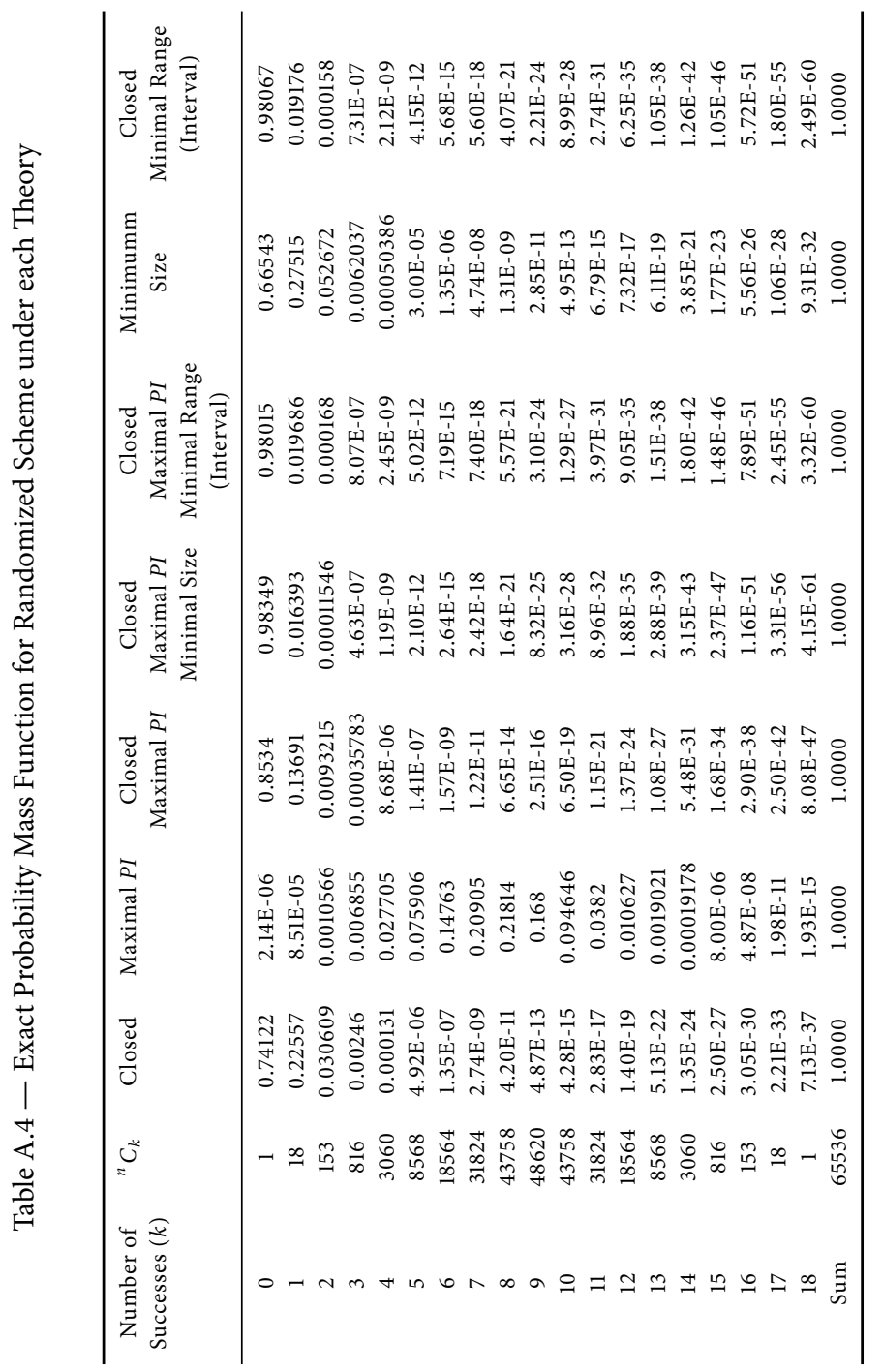




\section{Acknowledgements}

The authors wish to thank Matthew Braham, Moshé Machover, and Frank Steffen for their helpful comments. While working on this paper Dan Felsenthal was also co-director of the Voting Power and Procedures Programme at the Centre for Philosophy of Natural and Social Science, London School of Economics and Political Science. This programme is supported by Voting Power in Practice Grant F/07 004/AJ from the Leverhulme Trust.

\section{References}

Axelrod, R. (1970), Conflict of Interest: A Theory of Divergent Goals with Applications to Politics, Markham.

Chua, V.C.H. and D.S. Felsenthal (2008), Coalition formation theories revisited: an empirical investigation of Aumann's hypothesis, in: M. Braham and F. Steffen (eds.), Power, Freedom, and Voting: Essays in Honour of Manfred J. Holler, Springer, 159-183.

Coleman, J.S. (1971), Control of collectivities and the power of a collectivity to act, in: B. Lieberman (ed.), Social Choice, Gordon and Breach.

van Damme, E. (Interviewer) (1998), On the state of the art in game theory: An interview with Robert Aumann, Games and Economic Behavior 24: 181-210.

Felsenthal, D.S. and M. Machover (1998), The Measurement of Voting Power, Edward Elgar.

Felsenthal, D.S. and M. Machover (2000), Voting power and parliamentary defections: The 1953-54 French national assembly revisited, paper presented at the Workshop on Game Theoretic Approaches to Cooperation and Exchange of Information with Economic Application, University of Caen, France, May 25-27, 2000, downloadable from http://eprints.lse.ac.uk/594/.

Felsenthal, D.S. and M. Machover (2002), Annexations and alliances: When are blocs advantageous a priori?, Social Choice and Welfare 19: 295-312.

Felsenthal, D.S. and M. Machover (2004), A priori voting power: What is it all about?, Political Studies Review 2: 1-23.

Felsenthal, D.S. and M. Machover (2005), Voting power measurement: A story of misreinvention, Social Choice and Welfare 25: 485-506.

Felsenthal, D.S. and M. Machover (2008), Further reflections on the expediency and stability of alliances, in: M. Braham and F. Steffen (eds.), Power, Freedom, and Voting: Esaays in Honour Manfred J. Holler, Springer, 37-53.

Gamson, W.A. (1961), A theory of coalition formation, American Sociological Review 26: 373-382.

Laver, M. and N. Schofield (1990), Multiparty Government: Politics of Coalitions in Europe, Oxford University Press.

Leiserson, M.A. (1966), Coalitions in Politics: A Theoretical and Empirical Study (mimeographed doctoral dissertation), Yale University

Penrose, L.S. (1946), The elementary statistics of majority voting, Journal of the Royal Statistical Society 109: 53-57. 
Penrose, L.S. (1952), On the Objective Study of Crowd Behavior, H.K. Lewis.

Riker, W.H. (1959), A test of the adequacy of the power index, Behavioral Science 4: 120-131.

Riker, W.H. (1962), The Theory of Political Coalitions, Yale University Press.

Ross, S.M. (2002), A First Course in Probability Theory (6th Edition). Upper Saddle River, Prentice Hall.

Shapley, L.S. (1953), A value for $n$-person games, in: H.W. Kuhn and A.W. Tucker (eds.), Contributions to the Theory of Games II (Annals of Mathematics Studies), Princeton University Press.

Shapley, L.S. and M.J. Shubik (1954), A method for evaluating the distribution of power in a committee system, American Political Science Review 48: 787-792.

Swaan, A. de (1973), Coalition Theories and Cabinet Formation: A Study of Formal Theories of Coalition Formation Applied to Nine European Parliaments After 1918, Elsevier. 